\title{
Personalization of Biomechanical Models for Early Detection of Disease in Arrhythmogenic Cardiomyopathy
}

\author{
Nick van Osta ${ }^{1}$, Feddo Kirkels ${ }^{2}$, Aurore Lyon ${ }^{1}$, Tijmen Koopsen ${ }^{1}$, Tammo Delhaas ${ }^{1}$, Maarten J \\ Cramer $^{2}$, Arco J Teske ${ }^{2}$, Joost Lumens ${ }^{1}$ \\ ${ }^{1}$ Dept. of Biomedical Engineering, Cardiovascular Research Institute Maastricht (CARIM), \\ Maastricht, The Netherlands; \\ ${ }^{2}$ Dept. of Cardiology, University Medical Center Utrecht, Utrecht, The Netherlands
}

\begin{abstract}
Arrhythmogenic cardiomyopathy $(A C)$ is an inherited cardiac disease clinically characterized by lifethreatening ventricular arrhythmias and progressive cardiac dysfunction. Current electrocardiographic and structural imaging methods fail to detect early-stage $A C$ related myocardial disease in mutation carriers. Here, we propose a cardiac imaging-based personalized modelling approach that enables the identification and characterization of regional electro-mechanical tissue abnormalities in the vulnerable right ventricular $(R V)$ free wall of AC mutation carriers.

$R V$ tissue deformation data from 2 controls and 8 mutation carriers, covering various stages of AC disease, were used to personalize the CircAdapt model of the human heart and circulation. This resulted in estimates of contractility and stiffness in the apical, midventricular, and basal segments of the $R V$.

Apex-to-base heterogeneity in tissue properties, with increased stiffness and decreased contractility in the $R V$ basal region, was found in most patients and was largest in late-stage AC disease. Future studies should evaluate whether early-stage tissue heterogeneity is predictive for arrhythmic events or AC disease progression.
\end{abstract}

\section{Introduction}

Arrhythmogenic Cardiomyopathy (AC) is considered as an inherited cardiomyopathy predisposing to ventricular arrhythmias, sudden cardiac death (SCD), and more rarely (bi)ventricular dysfunction and heart failure. A genetic mutation is found in up to $60 \%$ of probands, mostly affecting desmosomal genes [1]. To prevent sudden cardiac death in these apparently healthy AC mutation carriers, early detection of pro-arrhythmic tissue substrates is important. Current conventional electrocardiographic and structural imaging methods lack sensitivity to detect early-stage AC-related myocardial disease in genotype-positive AC family members.
In a recent study [2], Mast et al. showed that regional right ventricular (RV) deformation abnormalities exist in most individuals with a pathogenic plakophillin-2 (PKP2) or desmoglein-2 mutation, even in those classified as subclinical (concealed) or electrical stage AC patients according to the 2010 International Task Force Criteria [3]. In the same study, generic patient simulations obtained with a biophysical model of the human heart and circulation suggested that those regional RV deformation abnormalities originate from regional contractile dysfunction either or not in combination with myocardial stiffening.

In a follow-up study [4], Mast et al. showed that earlystage AC mutation carriers with an abnormal pattern of basal RV deformation are more likely to progress into a more advanced $\mathrm{AC}$ disease stage than those with normal basal RV deformation. RV mechanical dispersion has also been reported in early stage AC patients [5] and has been found to be associated with arrhythmic outcome [6].

These observed regional differences in RV myocardial deformation suggest the existence of a detectable heterogeneity in local tissue properties in early-stage AC. A biophysical model of the human heart and circulation, such as CircAdapt (www.circadapt.org), describes the mechanistic link between myocardial tissue properties and regional myocardial deformation, based on wellestablished physics and physiology principles. We therefore hypothesize that cardiac imaging-based personalization of the CircAdapt model can reveal the severity of AC-related RV tissue disease. In this study, we aimed to develop such a patient-specific modelling approach and test its ability to characterize regional differences in RV myocardial tissue properties.

\section{Methods}

\subsection{Patient Study}

A total of 2 control subjects and 8 mutation carriers (4 male, age $33.8 \pm 14.9$ years) were selected from the AC 
cohort that was previously described in more detail [2]. Selection was based on covering the spectrum of AC disease severity. Longitudinal RV strain was measured in three segments (apex, mid, and base) using a standard echocardiographic deformation imaging protocol that has been described in more detail elsewhere [7]. LV and RV end-diastolic volumes $(154 \pm 26 \mathrm{~mL}$ and $184 \pm 38 \mathrm{~mL}$, respectively) and stroke volume $(85 \pm 21 \mathrm{~mL})$ were obtained by cardiac magnetic resonance imaging (CMR) performed on a 1.5-T scanner (Achieva, Philips Healthcare, Best, the Netherlands), according to standard AC protocol [8].

Based on their RV basal strain pattern, the subjects were classified in three subgroups, representing increasing severity of AC disease [3]. One mutation carrier and two controls had normal RV deformation (Type-I), four mutation carriers showed mildly abnormal deformation (Type-II), and three showed severely abnormal deformation (Type-III).

\subsection{Personalized Model Simulation}

The CircAdapt model was used to simulate cardiac pump function and cardiovascular system dynamics [9]. CircAdapt is a lumped-parameter model which couples regional myocardial tissue mechanics in the cardiac walls to pump mechanics and hemodynamics of a geometrically simplified four-chamber heart model and closed-loop circulation. Its MultiPatch module [10] was used to divide the RV free wall in three tissue segments, representing the basal, midventricular, and apical regions.

\subsubsection{Passive and Active Tissue Behavior}

Cardiac myofiber mechanics were modelled using a Hilltype contraction model including active (myofiber contraction) and passive (soft tissue deformation of the myocardium) stress components [10]. In this model, passive fiber stress is described by a nonlinear relation depending on sarcomere length $\left(l_{s}\right)$,

$$
\sigma_{f, p a s}=p_{1}\left(\left(\frac{l_{s}}{l_{s 0}}\right)^{p_{2}}-1\right)
$$

Table 1. Indices used for estimation of contractility and stiffness.

\begin{tabular}{lcl}
\hline Index & Definition \\
\hline TTPP & $\left(t_{P P, \text { model }}-t_{P P, \text { meas }}\right)$ & $/ 10 \mathrm{~ms}$ \\
PP & $\left(\max \left(\epsilon_{\text {model }}\right)-\max \left(\epsilon_{\text {meas }}\right)\right)$ & $/ 1 \%$ \\
TT50 & $\left(t_{50 \%, \text { model }}-t_{50 \%, \text { meas }}\right)$ & $/ 10 \mathrm{~ms}$ \\
TTPS & $\left(t_{\text {peak,model }}-t_{\text {peak,meas }}\right)$ & $/ 10 \mathrm{~ms}$ \\
ESS & $\left(\epsilon_{P V C, \text { model }}-\epsilon_{P V C, \text { meas }}\right)$ & $/ 1 \%$ \\
PS & $\left(\right.$ min $\left(\epsilon_{\text {model }}\right)-$ min $\left.\left(\epsilon_{\text {meas }}\right)\right)$ & $/ 1 \%$ \\
\hline TTPP: Time to peak pre-stretch; PP: Peak Pre-stretch; TT50: \\
Time to 50\% shortening; TTPS: Time to peak strain; ESS: End \\
Systolic Strain; PVC: Pulmonary Valve Closure; PS: Peak Strain \\
\hline
\end{tabular}

where $l_{s 0}$ represents stress-free fiber length, $p_{1}$ a scaling factor, referred to as stiffness in the remainder of this study, and $p_{2}$ the exponent determining the degree of non-linearity. We assumed $p_{2}$ and $l_{s 0}$ to be constant, i.e. 8.4 and $1.8 \mu \mathrm{m}$, respectively.

The active fiber stress is a function of time $(t)$, sarcomere length $\left(l_{s}\right)$, and contractile element length $\left(l_{s i}\right)$ multiplied by a scaling factor $a_{1}$, referred to as contractility in the remainder of this study.

$$
\sigma_{f, a c t}=a_{1} \text { function }\left(t, l_{s}, l_{s i}\right)
$$

\subsubsection{Parameter Estimation Protocol}

The CircAdapt model was personalized by fitting simulated RV strain patterns to the patient's RV strain patterns, since this region is typically first-affected. To that end, parameters related to regional RV stiffness and contractility, relative systole duration, and LV and RV cavity and wall volume were adjusted for each patient.

RV stiffness and contractility were estimated by minimizing the error between a set of simulated and measured RV strain indices, together capturing the pattern of regional RV deformation (Table 1 and Figure 1): time-to-peak-pre-stretch (TTPP), peak pre-stretch, time-to-50\%-shortening (TT50), time-to-peak-strain (TTPS), peak strain (PS), and end-systolic strain (ESS).

LV end-diastolic volume was fitted by changing the LV cavity volume while maintaining wall thickness constant. RV wall and cavity volume were changed to fit RV global longitudinal strain. Relative systolic duration was estimated from time to pulmonary valve closure.

To minimize the squared sum of error in the indices, the trust-region algorithm was used. The algorithm stopped when the estimated step size of the parameters became smaller than $0.1 \%$ of the original value. The regional tissue parameters described earlier were estimated in iterative steps until convergence to improve convergence of the parameter estimation algorithm.

To quantify the goodness of the fit, the unitless mean squared strain error (MSE) of the three segmental RV strain patterns was calculated as the squared sum of difference in strain between simulation and measurement, normalized to the cycle time.

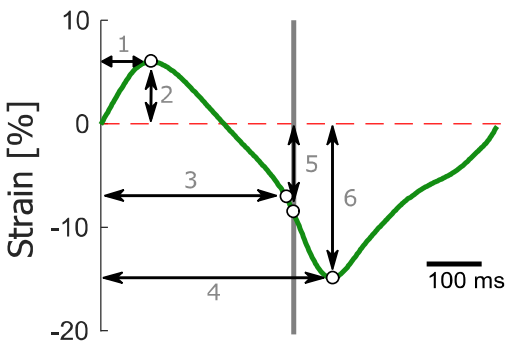

Figure 1. Indices used for fitting. Arrows represent the indices TTPP (1), Peak Pre-Stretch (2) TT50 (3), TTPS (4) End Systolic Strain (5), and Peak Strain (6), as described in Table 1. 


$$
M S E=\frac{\oint\left(\epsilon_{\text {model }}(t)-\epsilon_{\text {meas }}(t)\right)^{2} d t}{t \text { Cycle }}
$$

With $\epsilon_{\text {model }}$ and $\epsilon_{\text {meas }}$ being simulated and measured strain at time $t$, respectively. A perfect fit would result in an MSE of zero. The error is squared to increase the weight of larger differences in strain error. To quantify the degree of tissue heterogeneity for each type of AC disease, the standard deviation of the three segmental parameter values was calculated per subject and averaged for each subgroup.

\section{Results}

The simulated strains obtained from the personalized models are in good agreement with the measurements. The median MSE of all estimations is 2.6, with a minimum and maximum of 0.5 and 16 , respectively. The strain of two good and two bad estimations with MSE are shown in Figure 2. Figure 3 shows the mean estimated contractility and stiffness relative to the apical estimations for apical, mid, and basal segments. Both contractility and stiffness are more homogeneously distributed in Type-I, with a heterogeneity of $18 \%$ in contractility and a heterogeneity of $23 \%$ in stiffness. In Type-II and TypeIII, the parameters are more heterogeneously distributed with a heterogeneity of $23 \%$ in contractility and $83 \%$ in stiffness in Type-II, and a heterogeneity of $39 \%$ in contractility and $161 \%$ in stiffness in Type-III. On average, basal contractility decreases and stiffness increases for Type-II and Type-III, leading to an increase of $\mathrm{RV}$ tissue heterogeneity with progression of $\mathrm{AC}$ disease.

\section{Discussion}

The main outcome of this study is the characterization of the tissue mechanisms underlying deformation abnormalities in AC subjects using a patient-specific modeling-based approach. The results suggest that AC disease progression, defined as the level of RV basal deformation abnormality, is associated with growing RV apex-to-base heterogeneity in contractile dysfunction and myocardial stiffening. The patient-specific simulations of the subjects with a Type-II deformation pattern, which was previously found in about half of the subclinical mutation carriers, revealed decreased basal contractility and increased basal stiffness compared to the apex. This finding is in line with the hypothesis by Mast et al. [2].

These predicted changes in contractility and stiffness may be explained by fibro-fatty replacement, which is conventionally detected by late gadolinium enhancement (LGE). However, LGE was detected in only one Type-II and one Type-III patient. This suggests that our method may reveal insight in tissue abnormalities other than

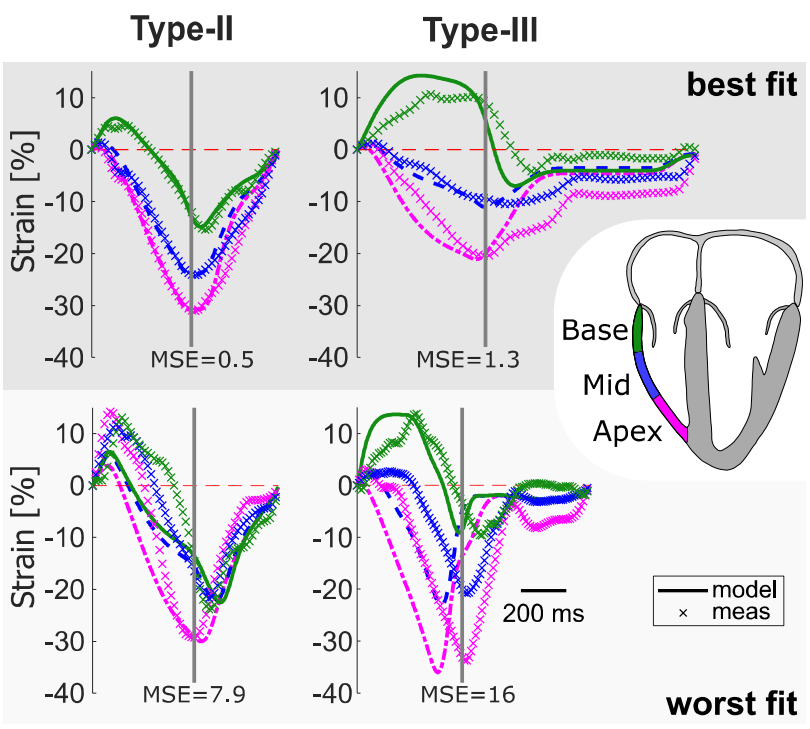

Figure 2. Two best (first and third from left) and two worst fits (second and fourth from left) in terms of MSE of Type-II and Type-III patients. The vertical line represents the closure of pulmonary valve in both measurements and model. Onset QRS is taken to be the zero-strain reference point.

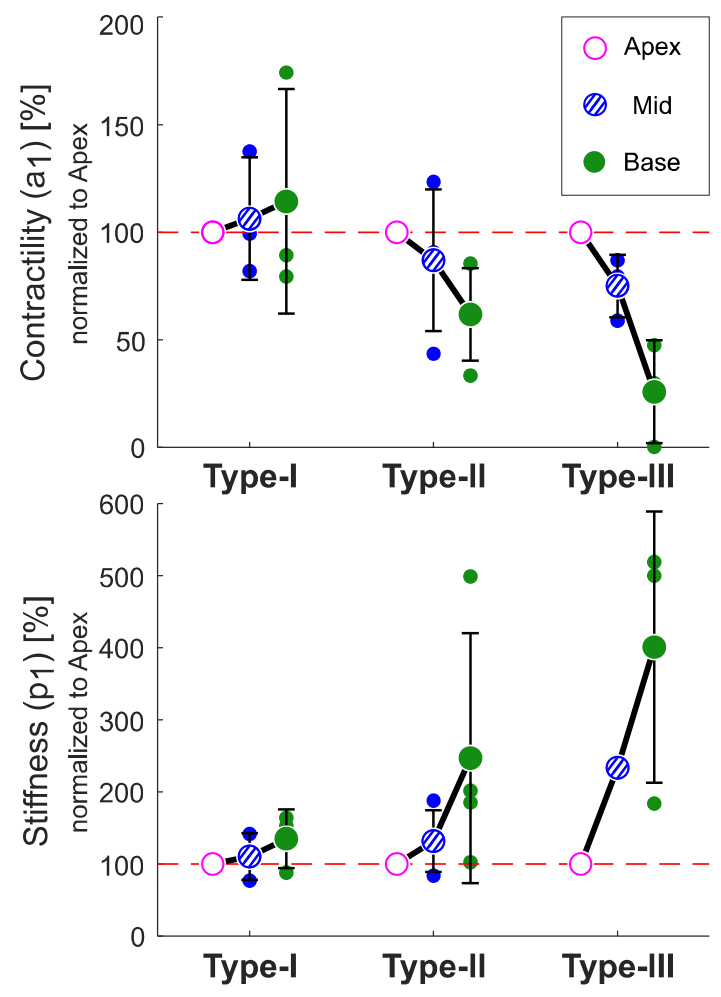

Figure 3. Fitted contractility and stiffness normalized to the apical values. The mean estimated values of contractility $a_{1}$ and stiffness $p_{1}$ per type (large dots) are shown with bars representing the standard deviation of the individual subjects (small dots). 
detected by LGE. This could also be in line with a limited sensitivity of LGE in the RV due to, for example, the typical thin RV lateral wall, and the presence of more diffuse fibrosis in AC patients [11].

Tissue behavior of the RV lateral wall depends not only on its intrinsic tissue properties, but also on mechanical interaction with the LV lateral and septal wall. This study focusses on the RV, because this is typically the first affected area in PKP2 mutation carriers [12]. In later disease stages, LV involvement can be observed at the macroscopic level [3], but also in early stages, as AC-related genes may be expressed differently in the LV [13]. Therefore, including LV tissue in the parameter estimation protocol might improve goodness of fit in the RV.

Currently, one fitting protocol with a fixed starting point is used. In the future, uniqueness of the solutions should be investigated. Future work can also develop the current estimation protocol by including other strain indexes to remove local minima, or by including other parameters to better capture the disease's mechanics. Finally, this study focuses on a relatively small cohort of PKP2 mutation carriers only. Future work should include a larger clinical sample size of a genetically more heterogeneous cohort of AC mutation carriers to evaluate the ability of the simulation based electromechanical substrates to stratify arrhythmic risk.

\section{Conclusion}

We presented a patient-specific modelling approach and showed its ability to reproduce regional RV deformation abnormalities in AC mutation carriers by introducing apex to base heterogeneity in regional tissue properties, more specifically a decreased basal contractility and an increased basal passive stiffness. Further research is needed to assess whether AC disease progression or arrhythmic events can be predicted by simulation-based characterization of early-stage disease substrates.

\section{Funding}

$\mathrm{NvO}, \mathrm{AL}$, and JL were funded through the Netherlands Organisation for Scientific Research (NWO-ZonMw, VIDI grant 016.176.340 to JL). TK and JL were funded by the Dutch Heart Foundation (2015T082 to JL). FK, TD, MJC, and AT received no specific funding for this work. The funders had no role in study design or data.

\section{References}

[1] Groeneweg JA, Bhonsale A, James CA, et al. Clinical Presentation, Long-Term Follow-Up, and Outcomes of 1001 Arrhythmogenic Right Ventricular Dysplasia/Cardiomyopathy Patients and Family
Members. Circ Cardiovasc Genet. 2015;8(3):437-46.

[2] Mast TP, Teske AJ, Walmsley J, et al. Right Ventricular Imaging and Computer Simulation for Electromechanical Substrate Characterization in Arrhythmogenic Right Ventricular Cardiomyopathy. J Am Coll Cardiol. 2016;68(20):2185-97.

[3] Marcus FI, McKenna WJ, Sherrill D, et al. Diagnosis of Arrhythmogenic Right Ventricular Cardiomyopathy/Dysplasia: Proposed Modification of the Task Force Criteria. Eur Heart J. 2010;31(7):80614.

[4] Mast TP, Taha K, Cramer MJ, et al. The Prognostic Value of Right Ventricular Deformation Imaging in Early Arrhythmogenic Right Ventricular Cardiomyopathy. JACC: Cardiovascular Imaging. 2018;

[5] Saberniak J, Leren IS, Haland TF, et al. Comparison of Patients with early-phase Arrhythmogenic Right Ventricular Cardiomyopathy and Right Ventricular Outflow Tract Ventricular Tachycardia. Eur Hear J Cardiovasc Imaging. 2017;18(1):62-9.

[6] Lie ØH, Rootwelt-Norberg C, Dejgaard LA, et al. Prediction of Life-Threatening Ventricular Arrhythmia in Patients with Arrhythmogenic Cardiomyopathy. JACC Cardiovasc Imaging. 2018;11(10):2660.

[7] Teske AJ, De Boeck BW, Melman PG, et al. Echocardiographic Quantification of Myocardial Function using Tissue Deformation Imaging, a Guide to Image Acquisition and Analysis using Tissue Doppler and Speckle Tracking. Vol. 5, Cardiovascular Ultrasound. BioMed Central; 2007. p. 27.

[8] Dalal D, Tandri H, Judge DP, et al. Morphologic Variants of Familial Arrhythmogenic Right Ventricular Dysplasia/Cardiomyopathy: A Genetics-Magnetic Resonance Imaging Correlation Study. J Am Coll Cardiol. 2009;53(15):1289-99.

[9] Arts T, Delhaas T, Bovendeerd P, et al. Adaptation to Mechanical Load Determines Shape and Properties of Heart and Circulation: the CircAdapt Model. Am J Physiol Heart Circ Physiol. 2005;288:1943-54.

[10] Walmsley J, Arts T, Derval N, et al. Fast Simulation of Mechanical Heterogeneity in the Electrically Asynchronous Heart Using the MultiPatch Module. PLoS Comput Biol. 2015;11(7):1-23.

[11] van Oorschot JWM, Güçlü F, de Jong S, et al. Endogenous Assessment of Diffuse Myocardial Fibrosis in Patients with T1 $\rho$-mapping. J Magn Reson Imaging. 2017;45(1):132-8.

[12] Teske AJ, Cox MGPJ, Te Riele ASJM, et al. Early Detection of Regional Functional Abnormalities in Asymptomatic ARVD/C Gene Carriers. J Am Soc Echocardiogr. 2012;25(9):997-1006.

[13] Asimaki A, Tandri H, Huang H, et al. A New Diagnostic Test for Arrhythmogenic Right Ventricular Cardiomyopathy. N Engl J Med. 2009;360(11):107584.

Address for correspondence:

Nick van Osta

Maastricht University

Universiteitssingel 50, P.O. Box 616, 6229 ER Maastricht

n.vanosta@maastrichtuniversity.nl 